\title{
Private and Social Incentives Towards Investment in Product Differentiation
}

\author{
Roberto Cellini* - Luca Lambertini** \\ * Dipartimento di Economia e Metodi Quantitativi \\ Università di Catania, Corso Italia 55, 95129 Catania, Italy \\ fax 39-095-370574, e-mail cellini@mbox.unict.it \\ ** Dipartimento di Scienze Economiche \\ Università degli Studi di Bologna \\ Strada Maggiore 45, 40125 Bologna, Italy
}

fax 39-051-2092664, e-mail lamberti@spbo.unibo.it

January 15, 2002

\begin{abstract}
We consider a dynamic oligopoly where firms invest to increase product differentiation and an externality effect operates in the R\&D activity. We compare the steady state solutions under alternative decision rules, namely, the open-loop and the closed-loop Nash equilibrium. Significant differences emerge, concerning the effect of the number of firms upon the optimal degree of product differentiation. We also compare the private optima with the social optimum, and derive implications concerning the social desirability of different decision rules.
\end{abstract}

J.E.L. Classification: C73, L13, O31

Keywords: differential games, R\&D investment, product differentiation, spillovers 


\section{Introduction}

We adopt a differential game approach to study the optimal effort of oligopolistic firms concerning the R\&D investment aimed at product innovation. In particular, we consider a dynamic model where firms produce symmetrically differentiated goods, and where the degree of differentiation is the outcome of R\&D investments by firms over time. Since the differentiation between goods is symmetric, it can be interpreted as a public good; thus, the activity of $\mathrm{R} \& \mathrm{D}$ aimed at product differentiation can be interpreted as the private provision of a public good.

The basics of the present model are the same as in Cellini and Lambertini (2002), but in the present paper we do not confine to the open-loop solution concept. On the contrary, we adopt the closed-loop Nash equilibrium concept. It is well-known that these two solution concepts may be interpreted as two different choice rules, corresponding to different information sets. Under the open-loop rule, firms precommit their decisions on the control variables to a path over time, and the resulting equilibrium is only (usually) weakly time-consistent. ${ }^{1}$ By contrast, under the closed-loop rule, firms do not precommit on any path and their strategies at any instant may depend on all the preceding history, even if -in such a situation- the information set used by firms in setting their strategies at any given time is often simplified to be only the current value of the state variables. The relevant equilibrium concept is the so-called "memoryless closed-loop" Nash equilibrium, and it is strongly time-consistent or Markov-perfect (see Başar and Olsder, 1982, and Dockner et al., 2000).

The comparison between the steady states under these different equilibrium concepts provides us with a view of some interesting properties of the optimal behaviour of oligopolistic firms under alternative decision rules. In particular, under the open-loop solution concept an Arrowian conclusion is reached, according to which the amount of resources invested by the industry in product differentiation is increasing in the number of firms, i.e., in the intensity of market competition (Arrow, 1962). By contrast, under the closed-loop time consistent solution concept, the Schumpeterian conclusion

\footnotetext{
${ }^{1}$ There exist classes of games where the open-loop is strongly time consistent. For an exhaustive overview, see Dockner et al. (2000, ch 7).
} 
may be reached, according to which the amount of resources invested by the industry in product differentiation is decreasing in the intensity of market competition, as captured by the number of firms (Schumpeter, 1942) .

Moreover, the privately optimal allocations under the aforementioned equilibrium concepts are compared with the social optimum. In particular, we show that the steady state equilibirum under the open-loop decision rules is closer to the steady state social optimum, than the closed-loop steady state equilibrium is.

The outline of the paper is as follows. Section 2 illustrates the basics of the model. Section 3 develops the private optimum under the closed-loop decision rule. Section 4 compares the results with the open-loop decision rules and derives comparison about profits and social welfare. Section 5 derives the social optimum allocation and proposes some considerations about the social desirability of market regulation under alternative private decision rules. Section 6 briefly concludes.

\section{The setup}

We consider a market where $n>1$ single-product firms sell differentiated products over the time period $(0, \infty)$. Market competition takes place à la Cournot. The demand structure is borrowed from Spence (1976). In each period $t \in(0, \infty)$, the inverse demand function for variety $i$ is:

$$
p_{i}(t)=A-B q_{i}(t)-D(t) \sum_{j \neq i} q_{j}(t)
$$

where $D(t) \in[0, B]$ is the symmetric degree of substitutability between any pair of varieties. If $D(t)=B$, products are completely homogeneous. If, on the contrary, $D(t)=0$, products are completely independent and each firm is an independent monopolist. ${ }^{2}$ At any time $t$, the output level $q_{i}(t)$ is produced at constant returns to scale, for a given $D(t)$, so that we define individual total operative cost per period as $C_{i}(t)=c q_{i}(t)$, where $c \in(0, A)$.

We assume that, at the initial instant $t=0, D(0)=B$, so that firms may produce the same homogeneous good through a technology which is

\footnotetext{
${ }^{2}$ The idea that $D$ depends upon the behaviour of firms has been investigated in static models by Harrington (1995); Lambertini and Rossini (1998); Lambertini, Poddar and Sasaki (1998).
} 
public domain. Product differentiation may increase, i.e., parameter $D$ may decrease, through firms' R\&D investment according to the following law, borrowed from Cellini and Lambertini (2002):

$\frac{d D(t)}{d t}=-\frac{K(t)}{1+K(t)} \cdot D(t) \equiv-\frac{k_{i}(t)+\sum_{j \neq i} k_{j}(t)}{1+\left[k_{i}(t)+\sum_{j \neq i} k_{j}(t)\right]} \cdot D(t) ; \quad k_{i}(t) \geq 0 \forall i$.

The above dynamics of product differentiation can be interpreted as follows. The industry overall R\&D expenditure is $K(t)$, while $k_{i}(t)$ is individual investment. Given the symmetric nature of product differentiation in this model, there exists a complete spillover effect in the R\&D process. Notice that the externality effect we consider here entails that the outcome of R\&D activity is public domain via the demand function. On the contrary, the externality effects usually considered in the literature are associated with information leakage or transmission (see, inter alia, d'Aspremont and Jacquemin, 1988). The dynamic equation (2) can be interpreted as a production function whose output is $-\left(\frac{\partial D(t)}{\partial t}\right) / D(t)$, obtained through an $\mathrm{R} \& \mathrm{D}$ input represented by capital. This technology can be shown to exhibit decreasing returns to scale w.r.t. $K(t)$. As a result, $D(t)$ is non-increasing over time, and approaches zero as $K(t)$ tends to infinity.

The instantaneous profit is $\pi_{i}(t)=\left[p_{i}(t)-c\right] q_{i}(t)-k_{i}(t)$. Each firm aims at maximizing the discounted value of its flow of profits:

$$
\Pi_{i}=\int_{0}^{\infty} e^{-\rho t} \pi_{i}(t) d t
$$

under the dynamic constraint (2) concerning the state variable $D(t)$. The control variables are $q_{i}(t)$ and $k_{i}(t)$.

\section{The private optimum}

Suppose firms choose non-cooperatively both R\&D efforts and output levels. The solution concept we adopt is the closed-loop memoryless Nash equilibrium. The objective function of firm $i$ is:

$$
\Pi_{i}=\int_{0}^{\infty} e^{-\rho t}\left\{q_{i}(t) \cdot\left[A-c-B q_{i}(t)-D(t) \sum_{j \neq i} q_{j}(t)\right]-k_{i}(t)\right\} d t
$$


to be maximised w.r.t. $q_{i}(t)$ and $k_{i}(t)$, under (2). The corresponding Hamiltonian function is:

$$
\begin{aligned}
\mathcal{H}(t)=e^{-\rho t} \cdot\left\{[A-c] q_{i}(t)-B\left(q_{i}(t)\right)^{2}-D(t) q_{i}(t) \sum_{j \neq i} q_{j}(t)-k_{i}(t)\right. \\
\\
\left.+\lambda_{i}(t)\left[-\frac{k_{i}(t)+\sum_{j \neq i} k_{j}(t)}{1+\left[k_{i}(t)+\sum_{j \neq i} k_{j}(t)\right]} \cdot D(t)\right]\right\},
\end{aligned}
$$

where $\lambda_{i}(t)=\mu_{i}(t) e^{\rho t}, \mu_{i}(t)$ being the co-state variable associated to $D(t)$.

The necessary and sufficient conditions for a path to be optimal are:

$$
\begin{gathered}
\frac{\partial \mathcal{H}(t)}{\partial q_{i}(t)}=A-c-2 B q_{i}(t)-D(t) \sum_{j \neq i} q_{j}(t)=0 \Rightarrow \\
q_{i}^{*}(t)=\frac{A-c-D(t) \sum_{j \neq i} q_{j}(t)}{2 B} ; \\
\frac{\partial \mathcal{H}(t)}{\partial k_{i}(t)}=-1-D(t) \lambda_{i}(t) \frac{1}{\left(1+k_{i}(t)+\sum_{j \neq i} k_{j}(t)\right)^{2}}=0 \\
-\frac{\partial \mathcal{H}(t)}{\partial D(t)}-\sum_{j \neq i} \frac{\partial \mathcal{H}(t)}{\partial q_{j}(t)} \frac{\partial q_{j}^{*}(t)}{\partial D(t)}-\sum_{j \neq i} \frac{\partial \mathcal{H}(t)}{\partial k_{j}(t)} \frac{\partial k_{j}^{*}(t)}{\partial D(t)}=\frac{d \mu_{i}(t)}{d t} \Rightarrow \\
\frac{d \lambda_{i}(t)}{d t}=q_{i}(t) \sum_{j \neq i} q_{j}(t)-\left[\sum_{j \neq i} D(t) q_{j}(t) \sum_{m \neq j} \frac{q_{m}(t)}{2 B}\right. \\
+\sum_{j \neq i} \frac{k_{2}\left[1+\lambda_{i}(t) \sqrt{-(t)} \frac{\left.\lambda_{i}(t)+\sum_{j \neq i} k_{j}(t)\right]^{2}}{\lambda_{i}(t) D(t)}\right.}{\lim _{t \rightarrow \infty}} \mu_{i}(t) \cdot D(t)=0 \\
\lambda_{i}(t)\left(\frac{k_{i}(t)+\sum_{j \neq i} k_{j}(t)}{1+\left[k_{i}(t)+\sum_{j \neq i} k_{j}(t)\right]}+\rho\right)
\end{gathered}
$$

We introduce the following: 
Assumption $q_{i}(t)=q_{j}(t)=q(t)$, and $k_{i}(t)=k_{j}(t)=k(t)$.

This is a usual symmetry assumption involving no loss of generality as long as one adopts the Nash equilibrium as the solution concept. In particular, it implies $\sum_{j \neq i} q_{j}(t)=(n-1) q(t)$ and $\sum_{j \neq i} k_{j}(t)=(n-1) k(t)$.

Then, from (6) we derive the equilibrium per firm output:

$$
q(t)=\frac{A-c}{2 B+(n-1) D(t)}
$$

which coincides with the standard outcome of Cournot models with product differentiation (see Singh and Vives, 1984; Majerus, 1988; Lambertini, 1997; Cellini and Lambertini, 1998, inter alia). Hence, given the implied symmetry condition $\lambda_{i}(t)=\lambda(t) \forall i,(7)$ rewrites as

$$
-\lambda(t)=\frac{[1+n k(t)]^{2}}{D(t)} .
$$

By symmetry, and using (11), (8) simplifies as follows:

$$
\begin{aligned}
\frac{d \lambda(t)}{d t}= & \frac{D(t)(n-1)[q(t)]^{2}[2 B-D(t)(n-1)]}{2 B D(t)}+ \\
& -\frac{B(1+n k)[2 n k+n-1+2 \rho(1+n k)]}{2 B D(t)} .
\end{aligned}
$$

From (11) we obtain $k(t)$, which can be differentiated w.r.t. $t$ :

$$
\frac{d k(t)}{d t}=\frac{1}{2 n \sqrt{-\lambda(t) D(t)}}\left[-\frac{d \lambda(t)}{d t} D(t)-\lambda(t) \frac{d D(t)}{d t}\right]
$$

Then, plugging (12) and (11)into $\partial k(t) / \partial t$ and rearranging, one obtains:

$$
\begin{aligned}
\frac{d k(t)}{d t} \propto & B[1+n k(t)][2 \rho(1+n k(t))+n-1]+ \\
& -D(t)(n-1)[q(t)]^{2}[2 B-D(t)(n-1)],
\end{aligned}
$$

since

$$
\frac{1}{2 n \sqrt{-\lambda(t) D(t)}}>0
$$


always. The expression in (14) is valid for all $D(t) \in(0, B]$. If $D(t)=0$, optimal per-period investment is $k(t)=0$. Likewise, one can also exclude the monopoly case $(n=1)$, where strategic interaction between goods is absent by definition. Therefore, in the remainder we focus on $n \geq 2$.

We are now in a position to assess the overall dynamic properties of the model, fully characterised by (13) and $d D(t) / d t=-n k(t) D(t) /(1+n k(t))$. The latter equation establishes that $d D(t) / d t<0$ for all $k(t) \in(0, \infty)$ and for all $D(t) \in(0, B]$; while $d D(t) / d t=0$ if $k(t)=0$ or $D(t)=0$. In the latter circumstances, it is immediate to verify that $\partial k(t) / \partial t$ is also nil.

Moreover, from (14), using the equilibrium value of the output level from (10), we obtain that

$$
\begin{gathered}
\frac{d k(t)}{d t}=0 \text { iff } \\
2 B \rho n^{2}[\alpha(t)]^{2}[k(t)]^{2}+B n[\alpha(t)]^{2}(4 \rho+n-1) k(t)+ \\
+B[\alpha(t)]^{2}(2 \rho+n-1)-(n-1)(A-c)^{2} D(t)[2 B-(n-1) D(t)]=0,
\end{gathered}
$$

where

$$
\alpha(t) \equiv 2 B+(n-1) D(t) .
$$

The roots of (16) are:

$$
\begin{aligned}
k(t)= & \frac{-B \cdot \alpha(t) \cdot(4 \rho+n-1) \pm \sqrt{\Omega}}{4 B \rho n \alpha(t)} \\
\Omega \equiv & 8(A-c)^{2} D(t) \alpha(t) \rho-B^{2}(n-1)[2 B-D(t)]^{2}+ \\
& +B n\left\{4 B[B+(n-2) D(t)]+[D(t)]^{2}\left(n^{2}-3 n+3\right)\right\} .
\end{aligned}
$$

The smaller root corresponds to a locus where $k$ has always negative values, and can therefore obviously be disregarded, being economically meaningless. Then, considering the larger root, we are interested in investigating the dynamics of the system in the positive quadrant of the space $\{D, k\}$, which is described in figure 1. The locus $d D(t) / d t=0$ corresponds to the axes. The locus $d k(t) / d t=0$ draws a curve over the admissible range of parameter $D$, which may or may not cross the horizontal axis within the same range, i.e., $D \in(0, B]$. If it does, the resulting candidate degree of substitutability in steady state is either

$D_{c l 1}=\frac{B\left[(A-c)^{2}-2 B(2 \rho+n-1)-(A-c) \sqrt{(A-c)^{2}-8 B(2 \rho+n-1)}\right]}{(n-1)\left[(A-c)^{2}+B(2 \rho+n-1)\right]}$, 
or

$D_{c l 2}=\frac{B\left[(A-c)^{2}-2 B(2 \rho+n-1)+(A-c) \sqrt{(A-c)^{2}-8 B(2 \rho+n-1)}\right]}{(n-1)\left[(A-c)^{2}+B(2 \rho+n-1)\right]}$.

with $D_{c l 1,2} \in \mathbb{R}$ iff $B \in(0, \bar{B}), \bar{B} \equiv(A-c)^{2} /[8(2 \rho+n-1)]$. Subscript $c l$ stands for closed-loop.

Moreover, it can be quickly checked that

$$
D_{c l 2}>D_{c l 1}>0
$$

in the whole admissible parameter range. Now there remains to check the conditions ensuring that $D_{c l 1,2}<B$.

It is easily established that $D_{c l 1}<B$ for all $n \geq 2$. As to the larger root, we have: ${ }^{3}$

$$
D_{c l 2}<B \text { for all } B>\widehat{B} \equiv \frac{(A-c)^{2}(n-3)(1-n)}{(n+1)^{2}(2 \rho+n-1)}
$$

with

$$
\begin{aligned}
& \bar{B}>\widehat{B}>0 \text { iff } n=2 ; \\
& \bar{B}>\widehat{B}=0 \text { iff } n=3 ; \\
& \bar{B}>0>\widehat{B} \text { for all } n \geq 4 .
\end{aligned}
$$

On the basis of the above discussion, we can formulate the following:

Lemma 1 If $n=2$ and $B \in(0, \widehat{B})$, there exists only one steady state at $D_{c l 1}$. If $n=2$ and $B \in(\widehat{B}, \bar{B})$, there exist two steady state levels of product substitutability, $D_{c l 1}$ and $D_{c l 2}$, with $B>D_{c l 2}>D_{c l 1}>0$. For all $n \geq 3$ and all $B \in(0, \bar{B})$, there exist two steady state levels of product substitutability, $D_{c l 1}$ and $D_{c l 2}$, with $B>D_{c l 2}>D_{c l 1}>0$.

${ }^{3}$ The equation $D_{1}^{*}=B$ has another root:

$$
B=-\frac{(A-c)^{2}}{2 \rho+n-1}
$$

which can be disregarded as it is always negative. 
The alternative situations identified by Lemma 1 are illustrated in figures $1-2$.

Figure 1 : Dynamics in the space $(D, k)$ for $n=2$ and $B \in(0, \widehat{B})$

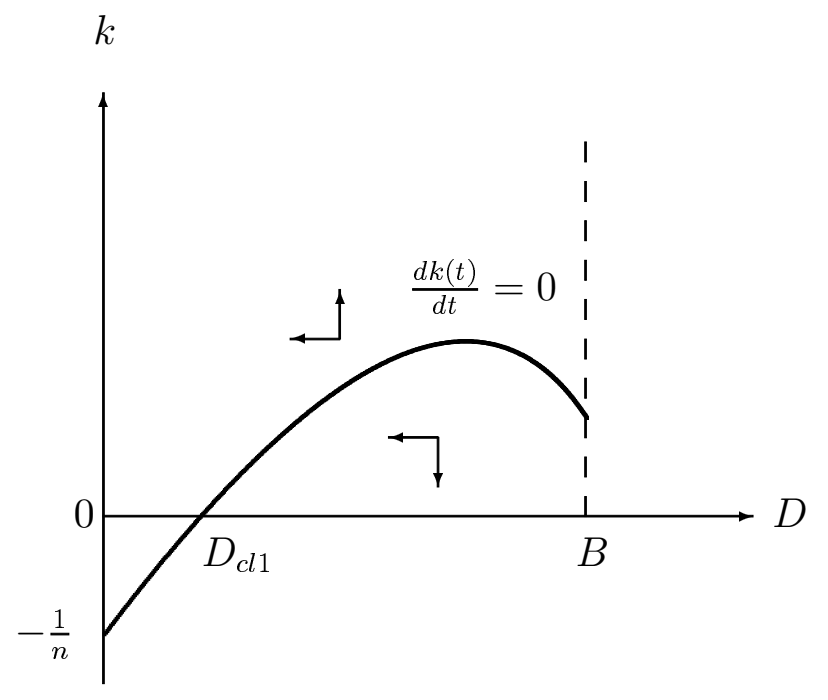


Figure 2 : Dynamics in the space $(D, k)$ for either

$$
\begin{aligned}
& \text { (i) } n=2 \text { and } B \in(\widehat{B}, \bar{B}) \text {, or } \\
& \text { (ii) } n \geq 3 \text { and all } B \in(0, \bar{B})
\end{aligned}
$$

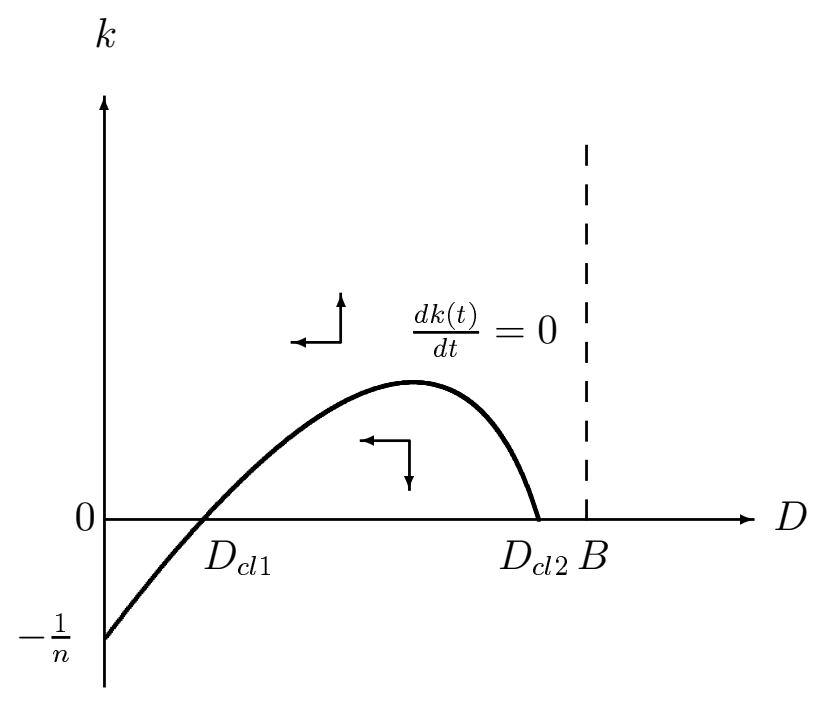

Considering the stability of the system, the system of dynamic equations (2) and (13) can be linearised around steady state points $\left(D_{c l 1}, 0\right)$ (whenever $\left.D_{c l 1} \in(0, B)\right)$ and $\left(D_{c l 2}, 0\right)$ to prove that the sign of the determinant of the Jacobian matrix is the sign of

$$
3 D(n-1)-2 B
$$

which

- in $\left(D_{c l 1}, 0\right)$ is negative for all $B \in(0, \bar{B})$;

- in $\left(D_{c l 2}, 0\right)$ is positive for all $B \in(0, \bar{B})$.

Since in $\left(D_{c l 2}, 0\right)$ the trace of the Jacobian matrix is positive over the whole admissible range of parameters, we can state the following: ${ }^{4}$

\footnotetext{
${ }^{4}$ The detailed calculations involved in the assessment of the stability properties are omitted for the sake of brevity. They are available from the authors upon request.
} 
Proposition 1 The steady state $\left(D_{c l 1}, 0\right)$ is always a saddle point. The steady state $\left(D_{c l 2}, 0\right)$, whenever it exists, is an unstable focus.

It remains to be stressed that the saddle point $\left(D_{c l 1}, 0\right)$ can obviously be approached only along the north-east arm of the saddle path.

\subsection{Comparative evaluation of open-loop and closed- loop equilibria}

Comparative statics exercises can be carried out on $D_{c l 1}$ to describe the effects of market size $(A-c)^{2} / B$, the discount rate $\rho$, and the number of firms $n$ on the optimal amount of product substitutability at the closed-loop equilibrium. In particular, the derivative $\partial D_{c l 1} / \partial n$ is a quartic expression in $n$, and can be evaluated only numerically, but a few examples suffice to characterise the behaviour of optimal substitutability as $n$ changes. For instance, consider the case $A-c=10$ and $\rho=1 / 10$. then, set $B=1 / 2$ to verify numerically that

$$
\begin{aligned}
& \frac{\partial D_{c l 1}}{\partial n}<0 \text { for all } n \in[2,4.3616) ; \\
& \frac{\partial D_{c l 1}}{\partial n}>0 \text { for all } n>4.3616 .
\end{aligned}
$$

In view of the integer constraint, this means that $D_{c l 1}$ is decreasing in $n$ for $n \in[2,4)$ and then increasing in $n$ for all $n \geq 5$. By increasing $B$, it can be seen that the interval wherein $\partial D_{c l 1} / \partial n<0$ shrinks. If $B=2.2425$, the closed-loop equilibrium degree of substitutability is the same at $n=2$ and $n=3$. $D_{c l 1}$ takes a minimum at $n=2.431$, where $D_{c l 1}=0.1297$. However, due to the integer constraint, the behaviour of $D_{c l 1}$ over $n \in[2,3)$ is irrelevant, and therefore if $B \in(2.2425, \bar{B})$, product differentiation is decreasing for all $n \geq 2$ at the closed-loop equilibrium. Of course this exercise can be repeated ad libitum for infinitely many admissible values of parameters $\{A, c, \rho\}$. In general, there exists a threshold $\widetilde{B}$ such that $D_{c l 1}$ is everywhere increasing in $n$ for all $B \in(\widetilde{B}, \bar{B})$.

That is, there exists a relevant parameter region where, whenever a real solution does exist, optimal product differentiation is decreasing in the number of firms at the closed-loop memoryless equilibrium. The intuition behind this result appears to be that, as the number of firms increases and the market 
becomes more competitive, the feedback effects characterising the closed-loop solution induce the generic firm to invest less in product differentiation in that, in the present model, differentiation is a public good whose benefits are becoming all the more difficult to internalise as $n$ grows larger.

In the light of the large debate on the relationship between market structure and the incentive to invest (either in process or in product innovation), that can be traced back to Schumpeter (1942) and Arrow (1962), ${ }^{5}$ these findings can be summarised by the following claim.

Proposition 2 Under the closed-loop information structure, an increase in market power generated by a decrease in the number of firms may lead to an increase in product differentiation at equilibrium.

That is, the closed-loop solution has a strong Schumpeterian flavour. This sharply contrasts with the conclusions drawn from the open-loop formulation of the same model.

From Cellini and Lambertini (2002), we know that the opposite holds at the open-loop equilibrium, where the optimal amount of product substitutability is:

$$
\begin{gathered}
D_{o l}=\frac{(A-c)^{2}-4 B \rho-(A-c) \sqrt{(A-c)^{2}-8 B \rho}}{2(n-1) \rho} \\
D_{o l} \in(0, B) \text { for all } B<\widetilde{B} \equiv \frac{(A-c)^{2}(n-1)}{\rho(n+1)^{2}}
\end{gathered}
$$

with subscript $o l$ standing for open-loop. Given that $\widetilde{B}>\bar{B}$ for all $n \geq 2$, both $D_{o l}$ and $D_{c l 1}$ belong to $(0, B)$ for all $B \in(0, \bar{B})$. Clearly, $\partial D_{o l} / \partial n<0$ always, i.e., the open-loop degree of product differentiation is increasing in the intensity of market competition. Increasing the number of firms reduces equilibrium profits, and this effect can be offset by enhancing R\&D efforts so as to decrease the degree of substitutability among products.

Then, comparing (20) and (26), it is straightforward to verify that

$$
D_{c l 1}-D_{o l}=0 \text { at } B_{1}=-\frac{(A-c)^{2}}{2 \rho+n-1} ; B_{2}=-\frac{(n-1)(A-c)^{2}}{(2 \rho+n-1)^{2}} ; B_{3}=0 \text {, }
$$

with $D_{c l 1}-D_{o l}>0$ for all $B>B_{3}$.

Consequently, the following holds:

\footnotetext{
${ }^{5}$ For an exhaustive overview of this literature, see Reinganum (1989).
} 
Proposition 3 Ceteris paribus, the difference $D_{c l 1}-D_{o l}$ is positive and increasing in $n$ for all $B \in(0, \bar{B})$.

This leads us to the comparative appraisal profits and of social welfare levels in the two settings. Concerning profits, observing that (i) optimal output (10) is the same under both open- and closed-loop information structure; and (ii) equilibrium investments in steady state are zero in both cases, suffices to prove that the following Corollary to Proposition 3 holds:

Corollary 1 Ceteris paribus, the individual firm's profits as well as industry profits, are lower in the closed-loop equilibrium than in the open-loop equilibrium.

This is a result that we are well accustomed with from the existing literature on dynamic investment in oligopoly, be such investment related to $\mathrm{R} \& \mathrm{D}$, productive capacity or other long run variables affecting firms' size (see, inter alia, Reynolds, 1987; and the related discussion in Fudenberg and Tirole, 1991, ch. 13).

As to the welfare assessment, we proceed as follows. The utility function of the representative consumer is:

$$
U(t)=A \sum_{i} q_{i}(t)-\frac{1}{2}\left[B \sum_{i=1}^{N}\left(q_{i}(t)\right)^{2}+D(t) \sum_{i} \sum_{j \neq i} q_{i}(t) q_{j}(t)\right]
$$

whose maximization under the budget constraint $Y(t) \geq \sum_{i} p_{i}(t) q_{i}(t)$, where $Y(t)$ is nominal income, yields demand functions (1). Accordingly, consumer surplus is measured by $C S(t) \equiv U(t)-\sum_{i} p_{i}(t) q_{i}(t)$ and social welfare is $S W(t) \equiv \sum_{i} \pi_{i}(t)+C S(t)$. Under the symmetry assumption, and given that $k=0$ in steady state, consumer surplus writes as

$$
C S=\frac{n q^{2}}{2}[B+D(n-1)]
$$

while welfare simplifies as follows:

$$
S W=n(A-c) q-\frac{n q^{2}}{2}[B+D(n-1)]
$$

Now it suffices to plug the equilibrium output (10) into (29) and (30) and differentiate w.r.t. $D$ to verify that both $\partial C S / \partial D$ and $\partial S W / \partial D$ are everywhere negative, which implies a second relevant Corollary to Proposition 3: 
Corollary 2 Ceteris paribus, social welfare is higher in the open-loop equilibrium than in the closed-loop equilibrium, due to both higher industry profits and higher consumer surplus.

If compared to most of the existing contributions in oligopoly theory, this result sounds quite peculiar. Usually, if firms' profits increase, consumer surplus decreases and the overall welfare effect is a priori ambiguous. In the present setting, however, given that first order conditions on output levels are the same under both solution concepts, an increase in product differentiation benefits both the firms and the consumers alike. I.e., what firms and consumers have in common, here, is a taste for differentiation ${ }^{6}$ which generates the above result.

\section{The social optimum}

The objective of a benevolent social planner consists in maximising the discounted value of social welfare under the dynamic constraint (2). Under the symmetry assumption, which in this setting can be imposed on outputs and R\&D efforts from the outset, the instantaneous social welfare simplifies as follows:

$$
S W(t)=n(A-c) q(t)-\frac{n[B+D(n-1)][q(t)]^{2}}{2}-n k(t)
$$

The Hamiltonian function corresponding to the maximisation of the present value of the social welfare is:

$$
\begin{gathered}
\mathcal{H}_{s p}(t)=e^{-\rho t} \cdot\left\{n(A-c) q(t)-\frac{n[B+D(t)(n-1)][q(t)]^{2}}{2}-n k(t)\right. \\
\left.-\frac{\lambda(t) D(t) n k(t)}{1+n k(t)}\right\},
\end{gathered}
$$

where the subscript $s p$ stands for social planning. The first order conditions and adjoint equations are:

$$
\frac{\partial \mathcal{H}_{s p}(t)}{\partial q(t)}=n(A-c)-n q(t)[B+D(t)(n-1)]=0 ;
$$

\footnotetext{
${ }^{6}$ It is worth stressing that consumers exhibit an additional taste for variety, that is, consumer surplus is increasing in the number of firms, $n$. This of course cannot hold for firms, as individual profits are decreasing in $n$.
} 


$$
\begin{gathered}
\frac{\partial \mathcal{H}_{s p}(t)}{\partial k(t)}=-n\left\{1+\frac{D(t) \lambda(t)}{[1+n k(t)]^{2}}\right\}=0 ; \\
-\frac{\partial \mathcal{H}_{s p}(t)}{\partial D(t)}=\frac{d \mu(t)}{d t} \Rightarrow \frac{d \lambda(t)}{d t}=\frac{n[q(t)]^{2}}{2}+\lambda(t)\left(\frac{n k(t)}{1+n k(t)}+\rho\right) ; \\
\lim _{t \rightarrow \infty} \mu(t) \cdot D(t)=0,
\end{gathered}
$$

with $\mu(t)$ being the costate variable associated with $D(t)$, and $\lambda(t)=\mu(t) e^{\rho t}$.

From (33) and (34), one obtains, respectively:

$$
\begin{gathered}
q(t)=\frac{A-c}{B+D(t)(n-1)} ; \\
-\lambda(t)=\frac{[1+n k(t)]^{2}}{D(t)} .
\end{gathered}
$$

The optimal quantity (37) reveals that, as one would clearly expect from the outset, the planner sets the price equal to marginal cost.

Again from (34), the evolution of $k(t)$ over time has the following features:

$$
\frac{d k(t)}{d t} \propto-\frac{d \lambda(t)}{d t} D(t)-\lambda(t) \frac{d D(t)}{d t} .
$$

Using (35), (37) and (38), one can rewrite the r.h.s. of the above expression as follows:

$$
\frac{d k(t)}{d t} \propto 2 \rho[1+n k(t)]^{2}-\frac{D(t) n(n-1)(A-c)^{2}}{[B+D(t)(n-1)]^{2}} .
$$

The roots of the r.h.s. of $(40)$ w.r.t. $k(t)$ are:

$$
k(t)=-\frac{1}{n} \pm \frac{(A-c) \sqrt{2 n(n-1) \rho D(t)}}{2 \rho n[B+D(t)(n-1)]} .
$$

Again, the smaller root can be disregarded as it is always negative. The larger root is zero at

$$
\begin{aligned}
& D_{s p 1}=\frac{n(A-c)^{2}-4 B \rho-(A-c) \sqrt{n\left[n(A-c)^{2}-8 B \rho\right]}}{4(n-1) \rho} ; \\
& D_{s p 2}=\frac{n(A-c)^{2}-4 B \rho+(A-c) \sqrt{n\left[n(A-c)^{2}-8 B \rho\right]}}{4(n-1) \rho},
\end{aligned}
$$


with

$$
D_{s p 1,2} \in \mathbb{R} \text { for all } B \in\left(0, \frac{n(A-c)^{2}}{8 \rho}\right] .
$$

Moreover, simple calculations are needed to check that

$$
\begin{aligned}
& D_{s p 1}>0 \text { always; } \\
& D_{s p 1}<B \text { for all } B>\frac{(n-1)(A-c)^{2}}{2 n \rho} ; \\
& D_{s p 2}>B \text { always. }
\end{aligned}
$$

Notice that

$$
\frac{n(A-c)^{2}}{8 \rho} \geq \frac{(n-1)(A-c)^{2}}{2 n \rho} \text { for all } n \geq 2 .
$$

We have thus proved the following:

Lemma 2 The social planner reaches a steady state at $D_{s p 1} \in(0, B)$ for all $B \in\left(\frac{(n-1)(A-c)^{2}}{2 n \rho}, \frac{n(A-c)^{2}}{8 \rho}\right)$.

This situation is illustrated in figure 3 .

Figure 3 : Dynamics in the space $(D, k)$ under social planning

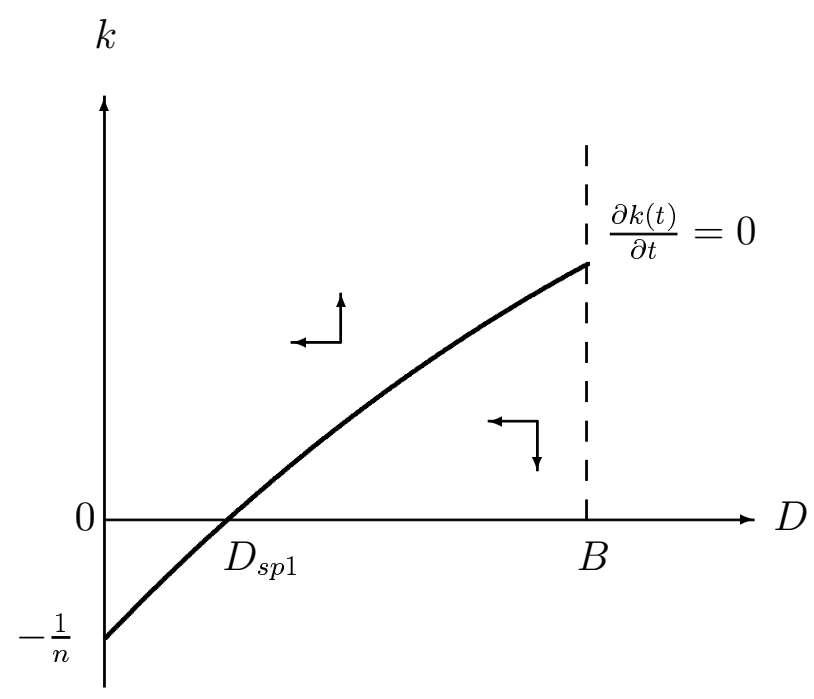


The dynamic properties of the system, as described by figure 3 , together with Lemma 2, suffice to prove the following:

Proposition 4 For all $B \in\left(\frac{(n-1)(A-c)^{2}}{2 n \rho}, \frac{n(A-c)^{2}}{8 \rho}\right)$, the socially optimal steady state $\left(D_{s p 1}, 0\right)$ is a saddle point, with each firm pricing at marginal cost.

Now evaluating $D_{s p 1}$ against $D_{o l}$ reveals that $D_{s p 1}<D_{o l}$ for all $B$ such that $D_{s p 1}, D_{o l} \in(0, B)$. The same holds if one compares $D_{s p 1}$ against $D_{c l 1}$. Hence we have our final result:

Corollary $3 D_{s p 1}<D_{o l}<D_{c l 1}$ for all $B \in(0, B)$. Hence, at the private optima product differentiation is too low as compared to social planning.

By increasing product differentiation as compared to the private optima, the planner gets two eggs in one basket, because welfare (which coincides here with consumer surplus) increases due to both an increase in consumer utility $U$, which is everywhere decreasing in $D$ for any given output level, and an increase in output levels (37).

In sharp contrast with the acquired wisdom (see, e.g., Tirole, 1988, ch. 7; and Eaton and Lipsey, 1989), in this model the private (i.e., firms') incentive to provide product differentiation is lower than the social incentive. ${ }^{7}$ This is due to the fact that, here product differentiation is a public good and therefore its benefits spill over completely to all the rivals, unlike what we see in the so-called address models where firms choose locations (as in the literature stemming from Hotelling, 1929) or qualities (as in Shaked and Sutton, 1982; 1983), where the internalised benefit exceeds the external effect.

\section{Concluding remarks}

We have taken a dynamic game approach to analyse firms' R\&D efforts to differentiate products. Under our hypotheses, differentiation is, at least

\footnotetext{
${ }^{7}$ We have shown this result by contrasting the fully private equilibria with the first best where the planner controls both output and investment levels. It could be shown that the same conclusion obtains in the case where firms control output levels while a public agency interested in welfare maximisation controls investment levels.
} 
partly, a public good. We have compared the open-loop Nash equilibrium strategy with the closed- loop (time-consistent) decision rule. We have shown that, under the closed-loop decision rule, multiple steady states may exist, but only one is a saddle. We have focused on this saddle point, and we have compared its properties with the properties of the saddle-point emerging from the open-loop Nash equilibrium.

Some results are worth stressing. First, the link between market competition, as captured by the number of firms in the market, and optimal efforts for product differentiation is different under the alternative decision rules. Indeed, under the open-loop equilibrium concept, a larger number of firms leads to a higher degree of differentiation, which in turn requires a higher aggregate effort. This conclusion has an anti-Schumpeterian flavour, lending support to the Arrowian idea that the harsher the competition, the higher the equilibrium level of R\&D. On the opposite, under the closed-loop equilibrium concept, when the number of firms is sufficiently large, a larger number of firms leads to a smaller degree of differentiation, requiring a smaller R\&D aggregate effort. This conclusion has a clear Schumpeterian flavour.

Second, the "period by period" reaction implied by the closed-loop decision rule leads to the result that the steady state individual firm's profits, as well as the steady state industry profits, are lower under the closed-loop that under the open-loop decision rule.

Moreover, the steady state degree of differentiation among goods under the open-loop is closer to the socially optimum level than the steady state degree of differentiation reached under the closed-loop rule. In both cases, the privately optimal product differentiation is too low as compared to social planning.

Finally, the social welfare in the steady state equilibrium under the closedloop decision rule is smaller that its counterpart under the open-loop rule, due to both higher industry profiuts and higher consumer surplus.

All the results are consistent with the fact that the differentiation is -in the present model- a public good. This assumption has led to the conclusion that the private incentive to produce differentiation is lower than the social incentive. 


\section{References}

[1] Arrow, K.J. (1962), "Economic Welfare and the Allocation of Resources for Invention", in R. Nelson (ed.), The Rate and Direction of Industrial Activity, Princeton, NJ, Princeton University Press.

[2] Basar, T. and G.J. Olsder (1982), Dynamic Noncooperative Game Theory, San Diego, Academic Press.

[3] Cellini, R. and L. Lambertini (1998), "A Dynamic Model of Differentiated Oligopoly with Capital Accumulation", Journal of Economic Theory, 83, 145-55.

[4] Cellini, R. and L. Lambertini (2002), "A Differential Game approach to Investment in Product Differentiation", Journal of Economic Dynamics and Control, forthcoming.

[5] Dockner, E.J, S. Jørgensen, N. Van Long and G. Sorger (2000), Differential Games in Economics and Management Science, Cambridge, Cambridge University Press.

[6] Eaton, B.C. and R.G. Lipsey (1989), "Product Differentiation", in R. Schmalensee and R.D. Willig (eds.), Handbook of Industrial Organization, Vol. 1, Amsterdam, North-Holland.

[7] Fudenberg, D. and J. Tirole (1991), Game Theory, Cambridge, Mass., MIT Press.

[8] Harrington, J.E. (1995), "Experimentation and Learning in a Differentiated-Products Duopoly", Journal of Economic Theory, 66, 275-88.

[9] Hotelling, H. (1929), "Stability in Competition", Economic Journal, 39, $41-57$.

[10] Lambertini, L. (1997), "Prisoners' Dilemma in Duopoly (Super)Games", Journal of Economic Theory, 77, 181-91.

[11] Lambertini, L. and G. Rossini (1998), "Product Homogeneity as a Prisoner's Dilemma in a Duopoly with R\&D", Economics Letters, 58, 297301. 
[12] Lambertini, L., S. Poddar and D. Sasaki (1998), "Standardization and the Stability of Collusion", Economics Letters, 58, 303-10.

[13] Majerus, D. (1988), "Price vs Quantity Competition in Oligopoly Supergames", Economics Letters, 27, 293-7.

[14] Reinganum, J. (1989), "The Timing of Innovation: Research, Development and Diffusion", in R. Schmalensee and R. Willig (eds.), Handbook of Industrial Organization, vol. 1, Amsterdam, North-Holland.

[15] Reynolds, S.S. (1987), "Capacity Investment, Preemption and Commitment in an Infinite Horizon Model", International Economic Review, 28, 69-88.

[16] Schumpeter, J.A. (1942, 2nd), Capitalism, Socialism and Democracy, New York, Harper.

[17] Shaked, A. and J. Sutton (1982), "Relaxing Price Competition through Product Differentiation", Review of Economic Studies, 69, 3-13.

[18] Shaked, A. and J. Sutton (1983), "Natural Oligopolies", Econometrica, 51, 1469-83.

[19] Singh, N. and X. Vives (1984), "Price and Quantity Competition in a Differentiated Duopoly", RAND Journal of Economics, 15, 546-54.

[20] Spence, A.M. (1976), "Product Differentiation and Welfare", American Economic Review, 66, 407-14.

[21] Tirole, J. (1988), The Theory of Industrial Organization, Cambridge, MA, MIT Press. 